\title{
Subjectively Experienced Time and User Satisfaction: An Experimental Study of Progress Indicator Design in Mobile Application
}

\author{
Sara Willermark \\ University West, School of \\ Business, Economics \& IT, Sweden \\ Sara.willermark@hv.se
}

\author{
Nikola Pantic \\ University West, School of \\ Business, Economics \& IT, Sweden \\ Nphemma@gmail.com
}

\author{
Hannah Pehrson \\ University West, School of \\ Business, Economics \& IT, Sweden \\ pehrson_hannah@hotmail.com
}

\begin{abstract}
In the user's interaction with systems, waiting and interruptions often constitute a source of negative experiences. However, system response time can be difficult or impossible to control. This study explores "subjective experienced time", which refers to the users' assessment of system response timeliness. The aim of this study is to gain increased knowledge of user satisfaction and subjectively experienced time in interaction with mobile applications. Thirty participants used and evaluated three mobile applications, containing unique stimuli in progress indicators. The results show correlation between progress indicators' degree of feedback and the subjectively experienced time and user satisfaction. Contributions include increased insight into the somewhat complex connection between the degree of feedback, subjectively experienced time and user satisfaction, as well as design implications for usercentred design.
\end{abstract}

\section{Introduction}

In the digitalized society, smartphones constitutes the most used personal devices [1] and for many people it is constantly accessible in the pocket $[2,3]$. It offers opportunities to work, communicate, run errands or simply get a moment of entertainment [4]. In that way the smartphone has become increasingly analogous to a "Swiss Army knife" as providing a plethora of readily-accessible tools for everyday life $[5,6]$. Interaction with computer systems always come with delays, due to for example poor internet connection, and has thus become part of smartphone users' everyday lives. Many studies have shown that system delays can have a great impact on user experience (UX) and performance [7-9]. When interacting with mobile applications, delays could be especially critical. Thus, on-the-go usage implies short and intensive interaction periods, which can hamper patience $[10,11]$. Furthermore, since mobile application shuts down when it is no longer handled in the processor it prevents the user from doing other activities while waiting. Research shows that we have a low tolerance for waiting and that users' tolerance for waiting in human-computer interactions gradually decreases. It has been reported that users can start to lose interest in the current task in waiting periods as short as two seconds [12, 13]. The best way to avoid suffering from waiting is clearly to reduce the actual delay time, as this shows a linear relationship with user satisfaction [14]. However, several technical factors such as browser performance, internet connection speed, local network traffic, and the web page structure are all related to the occurrence of delays [15]. Consequently, delays are not always possible to minimize. When the actual waiting time cannot be shortened, an alternative approach can be attempted to find ways to make users feel that time passes as quickly and pleasantly as possible [16]. Efforts to minimize design frictions i.e., difficulty occurring during interaction with technology, are often motivated from a desire to increase and maintain user engagement with a product [17].

However, there are also recent studies that suggests that design frictions not always perceived negatively by the user $[18,19]$. Instead, these user 'gaps' could be considered as opportunities that in fact disrupt "mindless" automatic interactions. This by prompting moments of reflection and cause more mindful interaction [17]. In summary, previous research reveals a complex picture of how to design for the benefit of the user experience. The aim of this study is to gain increased knowledge of user satisfaction and subjectively experienced time in interaction with 
mobile applications. The research question is as follows: "How does the design of progress indicators affects the user satisfaction and subjective experience of time in interaction with mobile applications?".

\section{Theoretical Framework and Related Research}

This section deals with theory and research about user satisfaction, subjectively experienced time and critical design approaches.

\subsection{User Experience and User Satisfaction}

In recent decades, User Experience (UX) has attracted the interest of both academics and industry practitioners. With increasing maturity of an industry, usability is more and more taken for granted [20]. Hence, it is not surprising that the concept of UX is widely discussed within the Human-Computer Interaction (HCI) community [21, 22]. There is little agreement regarding the exact notion of UX, yet many would agree that UX is about user satisfaction that goes beyond usability or problem solving [23]. Thus, the emergence of UX has drawn attention from functional, behavioural, and rational aspects of use [24] to affective, aesthetic, and phenomenological ones [25]. Moreover, researchers have come to expand the notion of user. From a goal-oriented performer, towards a more holistic view of a user as a person with feelings and preferences. Further, stresses the embodied, holistic experience of technology use which involves constant meaningmaking out of interaction [26]. Designing artefacts and services that put users' needs at the centre through a broad understanding of the users' goals and behaviours is considered increasingly important [27]. User experiences occur and are recalled over time. One account of experiences with technology emphasizes the continuous sensory connection with our environment situated in time that creates "felt life" as the ultimate experience [28].

In contrast, the prevailing cognitivist history of $\mathrm{HCI}$ research has typically reduced time to "system response time" (the time taken by the system to provide feedback for the previous user input) and "user response times" (the time that it takes for the user to provide new input after system feedback) [29]. This approach has been criticized by Liikkanen and Gómez [30] for being outdated and incompatible with recent UX debates. They argue that the psychological understanding of human time experience has not been truly utilized for HCI, nor articulated as design implications.

\subsection{Subjectively Experienced Time}

Experienced time is an elusive object of study. We all feel that we have a time sense, but this is certainly not a sense like the others [31]. Time perception is an integral part of psychological experience. Yet, since the duration of most meaningful experiences outlasts the capacity of working memory, people often have difficulty estimating how long experiences lasted [32]. Empirical studies suggest that duration estimates are influenced by many factors. It includes attentional engagement [33], arousal [34, 35] and motivation [36, 37].

Consequently, subjective duration often diverges from objective duration, and when this occurs time feels distorted. When time passes surprisingly quickly, it feels like time flew by and when time passes surprisingly slowly, it feels like time dragged on [32]. Previous research shows that experiences are evaluated positively when the passage of time is not noticed, or when no waiting is perceived. Thus, making people believe the time has flown by affects their enjoyment of a task, even if this belief is inaccurate $[32,38]$. In fact, when people believe that time has passed unexpectedly quickly, they rate tasks as more engaging, noises as less irritating, and songs as more enjoyable [32].

People do not perceive the passage of time in a linear way [39], instead there is a gap between perceived waiting time and actual waiting time [40]. This phenomenon makes it interesting to consider the experience of time in interaction with artefacts. It is well-known that emotions influence our behavior and thinking. For instance, when reflecting on past life we tend to remember emotional things better than neutral events [41, 42]. Furthermore, when recapping our experiences, we take their emotional peaks and final moments as representative of the whole [43]. In personal computing, momentary delays and malfunctioning seem commonplace. Consequently, our memories of interactions with computers can be biased by these negative experiences, often related to 
waiting [44]. Liikkanen and Gómez [30] introduce the concept of "subjectively experienced time" (hereafter SXT) to help design the way systems account for time. SXT is defined as the "assessment of system response timeliness" (p. 3), which refers to how the subjective response time makes the user feel. It is influenced by several factors, involving the users' past experiences as well as the present cognitive and affective state. Thus, SXT is individual and context dependent. Liikkanen and Gómez [26] argue that if users have learned that delays occur, in association with for example booking a ticket online for a popular concert or downloading a big file, this will influence their expectations. Consequently, even though the response time remains the same, the SXT is affected since the user can make sense of the delay and attribute it to an external source, thus influencing the subjective, emotional experience. Therefore, when designing for UX, SXT is of more relevance than the system response time and user response time. This is because SXT reflects the overall experience and extended waiting can provoke increasing levels of negative emotion, frustration and anger [30].

Meyer, Shinar [45] identified the importance of the progress indicator as a tool that enhance the attractiveness and effectiveness of programs that incorporate them, long ago. Furthermore, research has shown that in anticipation of loading sequences, it is important that the user is aware that the system is working, otherwise the user may experience a sense of lack of control. Feedback is necessary to capture the users' attention and communicate progress and it is of importance that the feedback provided is informative and correct $[12,27]$. Further, research suggest that users are most willing to tolerate negative progress behavior (e.g., stalls and inconsistent progress) at the beginning of an operation. However, some researchers claimed that more user feedback is not always better, as when people focus attention on temporal information, duration is perceived as longer [31, 45, 46]. In summary, previous research shows that it is essential to indicate that the system is working and that the information is correct. However, there is disagreement regarding how detailed the degree of feedback should be to minimize the SXT and promote user satisfaction, which is explored in this study.
There is previous research on progress indicators design. In a recent study, Kim, Xiong [47] explore online video viewers' perception of waiting time in relation to progress indicators. Each participant chose the best progress indicator in terms of SXT through a 7-point Likert scale. Their results showed that progress functions are important, as they are perceived as shorter than those of the repetitive and linear functions. However, the shape and embellishment of the progress indicator did not affect the results. Kurusathianpong and Tangmanee [48], explore the relationship between progress indicator design and SXT, in terms of graphics animation and length of the progress bar. The results provided empirical evidence of the impact between the size of progress bar and SXT, while the graphic animation had no effect. In contrast to these studies, the current study focus on interaction with hand-held digital devices (mobile applications), which can be considered particularly critical in terms of waiting time $[10,11]$.

However, in a recent study, Chen and $\mathrm{Li}$ [49] explored how visual feedback affected on users' perceptions of waiting time for a mobile application. More specifically, they explored three types of progress indicators (bar indicator, pie indicator, and cartoon indicator) effect on user's perception on time. The results suggest that the more complex designs (cartoon progress indicator) resulted in higher user satisfaction. However, while Chen and Li's [49] focuses on how the diversity of the design influences user experience, this paper focuses on how the degree of feedback provided influences SXT and user satisfaction.

\subsection{Critical design approaches}

There are several design traditions that seek to address the pervasive emphasis on effortless, efficient interaction, such as slow technology or reflective design [e.g. 50, 51]. Others have gone further and argued that technology can be designed to facilitate "uncomfortable interactions", where negative emotions server to enrich the UX [18, 19]. The aim is not about create long term discomfort or pain. Instead, the approach is based on the idea that many activities that make users uneasy are nonetheless valuable. This approach is particularly useful for drawing people's attention to important but difficult issues that they might naturally want to avoid. Critical design approaches promote reflection by 
subverting assumptions and expectations, often through making technology "unfriendly" to users [17, 52]. Thus, recent studies argue that designing friction into interactions can sometimes be justifiable and in fact have positive effect. Such "undesign" can be achieved through hindering particular interactions in order to promote reflection. Cox, Gould [17] argue for elicit such thoughtful interactions in a mild way. More specifically, they argue that there are times when it is motivated to design small frictions into interaction. Such approach offers a new way to think about improving everyday interactions with technology, by supporting a more effective digital behaviour. It does not mean advocating for design friction in their traditional sense and simply abandon principals for good design. Instead, the point of departure is that frictions that are designed with intention and introduced with care, have the potential to provoke interactions that are reflective and informed.

The line of argument derive from cognitive psychology [17, 53] and the assumption that human have two modes of though; System 1 respective System 2. System 1 constitutes the fast, automatic system that guides most of our behaviours and is employed during automatic and mindless interactions. System 2 constitutes the slower and more deliberate system that is employed when we are more mindful and conscious of what we are doing. By careful interaction design, System 2 could be invoked in a way that advantaged the user. Just one step in a procedure that takes slightly longer than necessary can provide an opportunity to avoid speed accuracy trade-offs in memory processes and thus increase accuracy; avoid being induced into performing behaviours that might not align with personal values, and; guide the user towards a particular course of desired action without having to rely on willpower alone. Such 'microboundary' is an intervention that provides a small obstacle prior to an interaction that prevents us rushing from one context to another. This by creating a brief moment in which the user might reflect on what they're doing. This small barrier to interaction can be implemented via a short time cost and prompts a switch from System 1 behaviour to that of System 2 [17]. Such approach contrasts with the idea of dark patterns of design which is about by making sure the users do not leave System1 [54].

\section{Method}

The overall research approach consists of a user test of mobile applications. Liikkanen and Gómez
[26] recommend guidelines for system development when designing for UX and SXT. This involves manipulating the user SXT and suggests that waiting could be turned into occupied time, by providing alternate tasks. However, such distractions can be difficult to realize when the user is handling a mobile device such as a smartphone, since the mobile application shuts down when it is no longer handled in the processor. This circumstance in combination with widespread and increasing use of smartphones makes it particularly interesting to study user satisfaction and SXT in smartphone usage. The study involved 1) a constructed user test; 2), an assessment questionnaire. This was conducted in the given order and during the same occasion. The participants were initially informed that they would test and evaluate an application "in the making", which was available in different versions. However, they were not informed of the difference between applications. They were asked to navigate within three applications and fill out a questionnaire for each application, which is elaborated below.

\subsection{Participants}

The study included 30 participants based on availability and willingness to participate. All participants attended a university in Sweden with an even distribution of men and women. Age (from 18 to 35 years old) and study affiliation varied. Participants were recruited through an open recruitment process, by advertising for participants on digital screens at the university as well as advertising on social media platforms connected to the university. The participants thereby willingly signed up for participation and were not targeted or approached specifically.

\subsection{User test and stimuli}

The purpose of the user test was to expose the participants to the independent variable (the progress indicator), and identify its possible effect on the dependent variables (user satisfaction and SXT). The experiment was designed so that all participants were exposed to three similar mobile applications. These applications were provided with different stimuli regarding progress indicators with different degree of feedback. The first ("repetitive progress indicator") offered a low degree of feedback. It provides information that the system is working by looping, yet does not give any information regarding how long the user will have to wait. The second ("linear progress indicator") offered more feedback than the first. It both provides information that the system is 
working and indicates how long an operation will take, as the animation gets filled. The third ("percent progress indicator") offered the most feedback. It provides information that the system is working and indicates how long an operation will take by "percent-done" animation (see Figure 1).

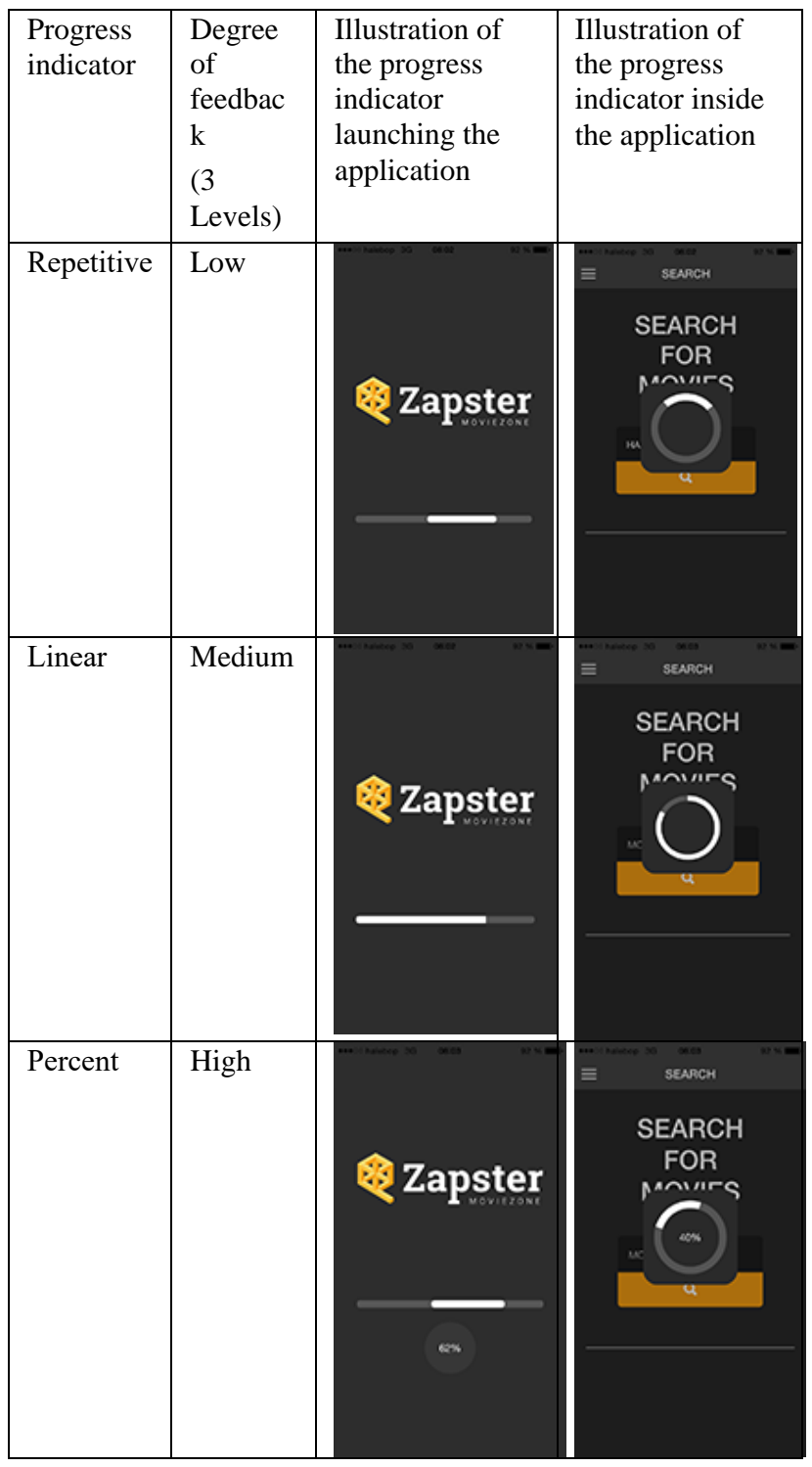

Figure 1. Experimental stimuli of the different progress indicators.

The stimuli were displayed three times within each application, i.e., 1) starting the application; 2) searching within the application; and 3) when loading a page. Each loading sequence was programmed to be displayed to participants for eight seconds per occasion. This was because durations shorter than five seconds often are considered "short" regardless of the differences among the loading symbols [47], and studies shows that users generally have a waiting tolerance threshold set to eight seconds, when interacting with interactive systems [13]. The participants were asked to interact with the applications according to identical instructions. By giving the participants a clear directive with a specific task to perform, the participants navigated within the applications and would be exposed to each stimulus.

All participants completed the test. Since the order of the interaction with the respective progress indicator could affect the participants' user satisfaction and SXT, there were three sets in which the order of applications varied, so that the participant was exposed to the applications in different orders, as follows;

a) 1. Repetitive progress indicator 2. Linear progress indicator, 3. Percent progress indicator

b) 1.Linear progress indicator, 2. Percent progress indicator, 3. Repetitive progress indicator

c) 1.Percent progress indicator, 2. Repetitive progress indicator, 3. Linear progress indicator

The participant was randomly assigned to the different sets. To isolate the independent variable, the applications were designed with an identical interface and content. The user test was carried out with a labowned device. The test was conducted at a secluded spot on campus. The aim was to recreate such as an authentic situation as possible, through the use of a familiar environment and realistic instructions with a reality-based purpose. Initially, we camouflaged the user test so that participants were not aware of its primary purpose and thus did not actively reflect on the perception of time [55]. In practice, this meant that we declared a vaguer purpose of the experiment as evaluating a prototype "in the making" and the participants were informed of the exact purpose of the experiment immediately after the test. Since it only constituted a subtle difference of purpose, it was not considered an ethical problem [55].

\subsection{Assessment questioner}

After interaction with each of the applications the participants were asked 1) to rate the overall impression of the application and, 2) to rate the "flow" of the application based on a 7-point Likert scale from "very well" to "very bad". This was 
conducted to capture the participants intuitive experience and thus serve as a "memory support" when comparing the applications in the assessment questionnaire. Immediately after interaction with all applications the participants were asked to fill out an assessment questionnaire where they were asked 1) to rank the applications based on preference 2) to rank the application based on waiting time, and 3) indicate how long the waiting time was perceived in each application (this constituted a control question to question number two).

\subsection{Analysis}

Ratings of SXT and user experience were collected and arranged for the statistical analysis. Significance of each factor effect on user satisfaction and SXT, and possible correlations between the variables was carried out. The results were analyzed using the nonparametric statistical Friedman's test.

\section{Results}

The results will be divided into three sections: 1) user satisfaction; 2) SXT and 3) correlation between the dependent variables. First, the difference in the material is tested to determine if there is any significance and if so, then the difference between the respective applications is tested.

\subsection{User satisfaction}

The "Percent" progress indicator was associated with the highest user satisfaction, followed by "Linear" and "Repetitive" (see Table 1). Three participants stated that they could not rank the applications according to satisfaction level. The test results show a high level of significance (Asymp. Sig. 0,02.), thus difference between applications was tested. The test results show that there is significance between "Repetitive" and "Linear" (Asymp. Sig. 0,04) and between "Repetitive" and "Percent" (Asymp. Sig.0,04). However, there is no statistically significant difference between "Linear" and "Percent" (Asymp. Sig. 0,336.).

\begin{tabular}{|l|l|l|l|}
\hline & "Repetitive" & "Linear" & "Percent" \\
\hline High & 4 & 10 & 13 \\
\hline Mid & 4 & 12 & 11 \\
\hline Low & 19 & 5 & 3 \\
\hline No opinion & \multicolumn{2}{|c|}{3} \\
\hline
\end{tabular}

Table 1. User satisfaction based on the different progress indicators $(\mathrm{N}=30)$

\subsection{SXT}

"Linear" progress indicator was the loading symbol that was perceived as the fastest, followed by "Percent" (see Table 2). "Repetitive" progress indicator was experienced as the slowest, which according to the participants was due to the lack of feedback regarding how much of the charge sequence remained. Three participants reported that they were unable to rate the loading symbols in relation to time experience as they reported that they did not experience any difference in the time span. Another participant stated that he experienced that repetitive was perceived as slowest, but was unable to rank the other two. The test results show that there is a high level of significance (Asymp. Sig. 0,028.) Although "Linear" was perceived as the fastest, it was not the most popular. Thus, the difference between the respective applications was tested. The test results show that there is significance between "Repetitive" and "Linear" (Asymp. Sig. 0,019) and "Repetitive" and "Percent" (Asymp. Sig. 0,050). However, there is no significance between "Linear" and "Percent" (Asymp. Sig. 0,695).

\begin{tabular}{|l|l|l|l|}
\hline & Repetitive & Linear & Percent \\
\hline Fastest & 5 & 12 & 9 \\
\hline Mid & 5 & 9 & 12 \\
\hline Slowest & 16 & 6 & 5 \\
\hline No opinion & 4 \\
\hline
\end{tabular}

Table 2: The degree of SXT based on the different loading symbols $(\mathrm{N}=30)$.

\subsection{Correlation between user satisfaction and SXT}

Thirteen of the participants reported a linear relationship between their SXT and their degree of user satisfaction due to the different stimuli they were 
exposed to, i.e., that the most satisfying stimulus was the same that was found to be the fastest and vice versa. However, 15 of the participants did not make this connection and two of the participants could not make any ranking.

\section{Discussion}

The results showed that low feedback as in "Repetitive" receives low rankings, both in terms of user satisfaction and SXT. This is in line with previous research that has shown the importance of feedback [12, 27, 47]. However, although "Repetitive" does indicate activity, it is not enough as revealed by the prevailing low ranking. Since several researchers have stressed the importance of feedback, these results are not particularly surprising. However, the ranking of "Linear" and "Percent" shows some rather complex results, as they receive the highest ranking in different aspects. Only half of the participants indicated a linear relationship between SXT and user satisfaction. This is in part contrary to the common perception of the connection between user satisfaction and SXT. However, it could be explained by the fact that when users tend to focus attention on temporal information, duration is perceived as longer [31, 45]. Thus, the detailed information may cause "Percent" to be perceived as somewhat slower. However, the fact that "Percent" is also the most appreciated in terms of user satisfaction suggests that it also shapes expectations [30], which contributes to user satisfaction, although in some cases it is perceived as bringing longer SXT. The results from this study suggest that the progress indicator affects the UX in terms of SXT and user satisfaction, yet does not provide a linear correlation. Instead, feedback seems to have a direct impact on user satisfaction without having to include SXT, although it can. The lack of clear results regarding "Linear" and "Percent" can also be explained by the tow designs being equivalent.

\subsection{Design implications}

When designing progress indicators, it is crucial to provide information about progress that extends beyond indicating that the system "is working". By providing feedback about progress, the users are not kept guessing, which has a positive impact on both SXT and user satisfaction.

However, the degree of feedback that should be provided is more complex. The results suggest that the progress indicator that resulted in the shortest SXT ("Linear") was not equated with the highest user satisfaction ("Percent"). This could pose a dilemma whether to prioritize SXT or user satisfaction in the selection of progress indicator. We argue that priority should be given to the progress indicator that promotes the highest user satisfaction. Thus, providing as detailed a level of information about the charging process as possible, although it may mean a longer SXT. As suggested by Cox, Gould [17] frictions are not always perceived negatively by the user [18, 19] but offers an opportunity for reflection and cause more mindful interaction [17]. From his perspective, waiting can in fact constitute a possibility in interaction with mobile applications, and facilitate the transition from System 1 to System 2. Such perspective directs interest in considering the design of micro-boundaries that offer an opportunity for reflection in a mild way. We argue that such perspective further reinforces the suggestion to prioritize user satisfaction over SXT, as a (perceived) delay can trigger reflection and mindfulness.

In this study, the simple design function of adjusting the degree of feedback in progress indicator effect user satisfaction and SXT with minimal adjustment. It can be considered in relation to Chen and $\mathrm{Li}$ [49] suggestions that more complex designs resulted in higher user satisfaction. From a deployment perspective, the findings of this paper are easier to be replicated and mass deployed in practice.

\subsection{Limitations and future work}

Even though this study focused on progress indicators in mobile applications, the results could be implemented in other human-computer interfaces. However, these results should be carefully applied in cases where the presentation setup differs greatly from ours or where the estimated waiting time differs greatly. The study has limitations that should be noted, and that also can serve as areas for future research. First, the experiment constitutes a constrained situation and thus direct application of study findings into real-life situations should be 
carried out with caution. However, measures were taken to create as authentic a situation as possible; the experimental setting constituted an environment that participants are familiar with and the activity carried out in the experiment was realistic. This was in order to create as representative a sequence of events as possible in an otherwise constructed situation [55]. Second, the stimuli of progress indicator were limited to eight seconds and a mobile application. It is therefore reasonable to consider that preferences could differ during another waiting time (as user experience may differ due to loading duration) or artefact (which allows the user to engage in other activities without hindering the loading of a page, etc.). Third, the data is limited and further studies are needed to determine results. Of interest is to further explore the relationship between feedback, user satisfaction and SXT. The results from this paper highlights the need for further research on the somewhat underestimated minor details of day-today interactions with technology. However, such studies should benefit from going beyond the labsetting, and out into real world "in-the-wild" interactions with technology. Further, research of interest includes the perception of time in different contexts, i.e., when the users are in an exploration mode versus task-oriented mode; or when the waiting time occurs in a leisure-based system versus a workoriented application; or when the users find themselves in a mobility situation, and thus can get external distraction during the wait.

\section{Conclusions}

This study shows that the degree of feedback in the progress indicator significantly affects user satisfaction and SXT related to charging sequences in mobile applications. Progress indicators that provide feedback in terms of progress functions were felt to be significantly shorter and brought more user satisfaction than the repetitive function only indicating activity. The results indicate that user satisfaction is promoted by a high degree of feedback (in percent), while users' SXT benefits from a slightly lower degree of feedback. The findings of this study contribute to the research field by suggesting that user satisfaction and SXT constitute a rather complex relationship, contrary to the common perception, and suggest design implications to create better loading symbols and human-computer interfaces rooted in user-centric design. Further, this study contributes to research on the 'mundane' and the details of day-to- day interactions with computers.

\section{References}

[1] Le, H.V., et al. Investigating screen shifting techniques to improve one-handed smartphone usage. in Proceedings of the 9th Nordic Conference on Human-Computer Interaction. 2016.

[2] Hinckley, K., et al. Sensing techniques for mobile interaction. in Proceedings of the 13th annual ACM symposium on User interface software and technology. 2000.

[3] Chen, X.A., et al. Duet: exploring joint interactions on a smart phone and a smart watch. in Proceedings of the SIGCHI Conference on Human Factors in Computing Systems. 2014.

[4] Smith, A. and M. Anderson, Social media use in 2018. Pew Research Center, 2018. 1.

[5] Böhmer, M., et al. Falling asleep with Angry Birds, Facebook and Kindle: a large scale study on mobile application usage. in Proceedings of the 13th international conference on Human computer interaction with mobile devices and services. 2011.

[6] Satyanarayanan, M., Swiss army knife or wallet? IEEE Pervasive Computing, 2005. 4(2): p. 2-3.

[7] Ceaparu, I., et al., Determining causes and severity of enduser frustration. International journal of human-computer interaction, 2004. 17(3): p. 333-356.

[8] Thum, M., et al., Standardized task strain and system response times in human-computer interaction. Ergonomics, 1995. 38(7): p. 1342-1351.

[9] Wilson, H.K. and A. Cotgrave, Factors that influence students' satisfaction with their physical learning environments. Structural Survey, 2016. 34(3): p. 256-275.

[10] Choi, J.H. and H.-J. Lee, Facets of simplicity for the smartphone interface: A structural model. International Journal of Human-Computer Studies, 2012. 70(2): p. 129142.

[11] Miniukovich, A. and A. De Angeli. Visual impressions of mobile app interfaces. in Proceedings of the 8th Nordic Conference on Human-Computer Interaction: Fun, Fast, Foundational. 2014.

[12] Nah, F.F.-H., A study on tolerable waiting time: how long are web users willing to wait? Behaviour \& Information Technology, 2004. 23(3): p. 153-163.

[13] Institute, S.B.R., Attention Span Statistics. 2015: https://www.statisticbrain.com/attention-span-statistics/.

[14] Fischer, A.R., F.J. Blommaert, and C.J. Midden, Monitoring and evaluation of time delay. International Journal of Human-Computer Interaction, 2005. 19(2): p. 163-180.

[15] Branaghan, R.J. and C.A. Sanchez, Feedback preferences and impressions of waiting. Human factors, 2009. 51(4): p. 528-538.

[16] Pruyn, A. and A. Smidts, Effects of waiting on the satisfaction with the service: Beyond objective time 
measures1. International journal of research in marketing, 1998. 15(4): p. 321-334.

[17] Cox, A.L., et al. Design frictions for mindful interactions: The case for microboundaries. in Proceedings of the 2016 CHI Conference Extended Abstracts on Human Factors in Computing Systems. 2016.

[18] Benford, S., et al. Uncomfortable interactions. in Proceedings of the sigchi conference on human factors in computing systems. 2012.

[19] Fokkinga, S.F. and P.M. Desmet, Ten ways to design for disgust, sadness, and other enjoyments: A design approach to enrich product experiences with negative emotions. International Journal of Design, 2013. 7(1).

[20] Alves, R., P. Valente, and N.J. Nunes. The state of user experience evaluation practice. in Proceedings of the 8th Nordic Conference on Human-Computer Interaction: Fun, Fast, Foundational. 2014.

[21] Vermeeren, A.P., et al. User experience evaluation methods: current state and development needs. in Proceedings of the 6th Nordic Conference on HumanComputer Interaction: Extending Boundaries. 2010. ACM.

[22] Vitiello, G., et al. UX-Requirements for Patient. in 2017 IEEE 25th International Requirements Engineering Conference Workshops (REW). 2017. IEEE.

[23] Kuutti, K. Where are the Ionians of user experience research? in Proceedings of the 6th Nordic conference on human-computer interaction: extending boundaries. 2010. ACM.

[24] Hassenzahl, M. and N. Tractinsky, User experience-a research agenda. Behaviour \& information technology, 2006. 25(2): p. 91-97.

[25] Norman, D.A., Emotional design: Why we love (or hate) everyday things. 2004: Basic Civitas Books.

[26] Wright, P. and J. McCarthy. Empathy and experience in HCI. in Proceedings of the SIGCHI Conference on Human Factors in Computing Systems. 2008. ACM.

[27] Norman, D., The design of everyday things: Revised and expanded edition. 2013: Constellation.

[28] McCarthy, J. and P. Wright, Technology as experience. interactions, 2004. 11(5): p. 42-43.

[29] Dabrowski, J. and E.V. Munson, 40 years of searching for the best computer system response time. Interacting with Computers, 2011. 23(5): p. 555-564.

[30] Liikkanen, L.A. and P.G. Gómez. Designing interactive systems for the experience of time. in Proceedings of the 6th International Conference on Designing Pleasurable Products and Interfaces. 2013. ACM.

[31] Macar, F., S. Grondin, and L. Casini, Controlled attention sharing influences time estimation. Memory \& cognition, 1994. 22(6): p. 673-686.

[32] Sackett, A.M., et al., You're having fun when time flies: The hedonic consequences of subjective time progression. Psychological Science, 2010. 21(1): p. 111-117.

[33] Chaston, A. and A. Kingstone, Time estimation: The effect of cortically mediated attention. Brain and Cognition, 2004. 55(2): p. 286-289.

[34] Campbell, L.A. and R.A. Bryant, How time flies: a study of novice skydivers. Behaviour research and therapy, 2007. 45(6): p. 1389-1392.

[35] Kellaris, J.J. and S.P. Mantel, Shaping time perceptions with background music: The effect of congruity and arousal on estimates of ad durations. Psychology \& Marketing, 1996. 13(5): p. 501-515.

[36] Conti, R., Time flies: Investigating the connection between intrinsic motivation and the experience of time. Journal of personality, 2001. 69(1): p. 1-26.

[37] Vohs, K.D. and B.J. Schmeichel, Self-regulation and extended now: Controlling the self-alters the subjective experience of time. Journal of personality and social psychology, 2003. 85(2): p. 217.

[38] Blonder, R., et al., Can You Tube it? Providing chemistry teachers with technological tools and enhancing their selfefficacy beliefs. Chemistry Education Research and Practice, 2013. 14(3): p. 269-285.

[39] Allan, L.G., The perception of time. Perception \& Psychophysics, 1979. 26(5): p. 340-354.

[40] Jones, P. and E. Peppiatt, Managing perceptions of waiting times in service queues. International Journal of Service Industry Management, 1996. 7(5): p. 47-61.

[41] Bradley, M.M., et al., Remembering pictures: pleasure and arousal in memory. Journal of experimental psychology: Learning, Memory, and Cognition, 1992. 18(2): p. 379.

[42] Buchanan, T.W. and W.R. Lovallo, Enhanced memory for emotional material following stress-level cortisol treatment in humans. Psychoneuroendocrinology, 2001. 26(3): p. 307-317.

[43] Fredrickson, B.L., Extracting meaning from past affective experiences: The importance of peaks, ends, and specific emotions. Cognition \& Emotion, 2000. 14(4): p. 577-606.

[44] Lazar, J., A. Jones, and B. Shneiderman, Workplace user frustration with computers: An exploratory investigation of the causes and severity. Behaviour \& Information Technology, 2006. 25(03): p. 239-251.

[45].Meyer, J., et al., Duration estimates and users' preferences in human-computer interaction. Ergonomics, 1996. 39(1): p. 46-60.

[46] Lallemand, C. and G. Gronier. Enhancing User eXperience during waiting time in HCI: contributions of cognitive psychology. in Proceedings of the Designing Interactive Systems Conference. 2012. ACM.

[47] Kim, W., S. Xiong, and Z. Liang, Effect of Loading Symbol of Online Video on Perception of Waiting Time. International Journal of Human-Computer Interaction, 2017. 33(12): p. 1001-1009.

[48] Kurusathianpong, P. and C. Tangmanee. Comparison of Perceived Waiting Time Between Two Lengths of Progress Indicator and Two Styles of Graphics Animation With Perceived Uncertainty as a Covariate. in 2018 Seventh ICT International Student Project Conference (ICT-ISPC). 2018. IEEE.

[49] Chen, C.-H. and S. Li, The effect of visual feedback types on the wait indicator interface of a mobile application. Displays, 2020. 61: p. 101928.

[50] Redström, J., et al., Slow Technology-Designing for Reflection. Personal Ubiquitous Comput, 2001. 5(3): p. 201-212.

[51] Sengers, P., et al. Reflective design. in Proceedings of the 4th decennial conference on Critical computing: between sense and sensibility. 2005.

[52] Dunne, A., Hertzian tales: Electronic products, aesthetic experience, and critical design. 2008, Royal College of Art. 
[53] Daniel, K., Thinking, fast and slow. Allen Lane, New York, USA, 2011.

[54] Brignull, H., Dark Patterns: Deception vs. Honesty in UI Design. Interaction Design, Usability, 2011. 338.

[55] Bryman, A., Social research methods. 2015: Oxford university press. 